\title{
The Effect of Chromium Picolinate Supplementation on the Pancreas and Macroangiopathy in Type II Diabetes Mellitus Rats
}

\author{
Shan Huang, Wenfang Peng, Xiaohong Jiang, Kan Shao, Lili Xia, \\ Yubin Tang, and Jiayin Qiu
}

Shanghai Tong Ren Hospital Affiliated to Shanghai Jiaotong University Medical School, Xianxia Road No. 1111, Changning District, Shanghai 200336, China

Correspondence should be addressed to Shan Huang; shanhuangsh@163.com

Received 27 February 2014; Revised 22 April 2014; Accepted 22 April 2014; Published 25 June 2014

Academic Editor: Aristidis Veves

Copyright (C) 2014 Shan Huang et al. This is an open access article distributed under the Creative Commons Attribution License, which permits unrestricted use, distribution, and reproduction in any medium, provided the original work is properly cited.

Purpose. The aim was to explore the effect of the chromium picolinate $(\mathrm{CrPic})$ administration on the pancreas and macroangiopathy of type II diabetes mellitus rats. Methods. The type II diabetes mellitus (T2DM) rat model was induced by low-dose streptozotocin (STZ). The rats were randomly divided into 5 groups (ten rats in each group). After supplementing CrPic for 15 weeks, the histopathological examination was performed by hematoxylin-eosin (HE) staining. Serum insulin and NO level were determined by radioimmunoassay and colorimetry, respectively. Serum glycosylated hemoglobin (HbA1C), adiponectin (APN), advanced glycation end products (AGES), and apelin were measured by ELISA. Real-time reverse transcription polymerase chain reaction (RT-PCR) was applied for detecting the mRNA expression of APN and apelin. Results. After CrPic treatment, compared with the T2DM control group (group 2), pancreas sections stained with HE showed the completed pancreatic cells structure and no inflammatory infiltration in groups 4 and 5. In addition, the levels of serum $\mathrm{NO}$ and insulin were significantly increased and the serum levels of $\mathrm{HbA1C}$, AGES, APN, and apelin were significantly decreased in groups 4 and 5 compared with group 2 . The mRNA expression of $A P N$ and apelin in groups 4 and 5 was also recovered to the normal level. Conclusion. CrPic can recover the function of B-cells and alleviate macroangiopathy in STZ-induced T2DM rats.

\section{Introduction}

Diabetes mellitus (DM) is one kind of metabolic syndromes characterized by chronic hyperglycemia [1]. The cases of diabetes fall into two broad pathogenetic categories: type I and type II diabetes mellitus (T1DM and T2DM). T2DM, which is previously referred to as noninsulin-dependent diabetes mellitus (NIDDM), accounts for approximately $90 \%$ of the diabetes patients $[2,3]$. There were many complications, such as nephropathy [4], cataracts [5], microangiopathy, and macroangiopathy [6] in T2DM. Among them, macroangiopathy is the most frequent complication in the patients with T2DM [7-9], which manifests as atherosclerosis like in nondiabetic patients and is characterized by formation of plaques that follows in stages but with an accelerated course due to the several risk factors [10].

In current clinical treatment, western medicines are widely used to control the hyperglycemia, hyperlipidemia, and insulin resistance of type 2 diabetes mellitus, such as sulfonylurea, biguanides, thiazolidinediones, and glycosidase inhibitors [11]. However, the clinical efficacy of these drugs in treating T2DM is limited. Thus, it is urgently needed to explore the novel drugs and therapies to improve the treatment effect and reduce the risk of complications of T2DM.

Chromium (Cr), as an essential element, is directly related to the activity of glucose tolerance factor (GTF) [12]. Cr can alleviate glucose intolerance and insulin resistance [13] and it is involved in the metabolism of glucose, lipid, protein, and nucleic acid [14-17]. However, as supplementary drug, $\mathrm{Cr}$ could not be effectively used due to the poor absorption rate (dietary chromium: $0.4-2 \%$; chromium chloride: $0.5-2 \%$ ) [18-20]. Chromium picolinate (CrPic), also named as picolinic acid chromium, is a convenient form of chromium that is used more efficiently than some other forms of chromium [10]. The absorption rate of it is about $0.7-5.2 \%$ [19]. Several 
studies have proved that $\mathrm{CrPic}$, as the source of $\mathrm{Cr}$, can alleviate the high level of blood glucose, blood lipid, insulin, and cholesterol in the patients with metabolic syndrome $[10,21,22]$. Therefore, the efficacy of CrPic in treating T2DM is undisputed. However, the efficacy of CrPic in treating macroangiopathy in patients with T2DM has not been known.

In the present study, we evaluated the effect of $\mathrm{CrPic}$ on pancreas and macroangiopathy in T2DM rat model by detecting the levels of serum and fat markers of T2DM. The model was induced by streptozotocin (STZ).

\section{Materials and Methods}

2.1. Animals and Diets. Male Wistar rats $(n=50)$ weighting $250 \pm 20 \mathrm{~g}$ were purchased from the animal center of Chinese academy of science. The animals were reared in a specific pathogen-free (SPF) laminar flow cabinet with temperature of $25 \pm 1^{\circ} \mathrm{C}$, humidity of $40-60 \%$, and a $12: 12 \mathrm{~h}$ light-dark cycle. The conventional diet and sterilized water were used to feed these rats.

The conventional diet and the high fat diet (HFD) were purchased from the laboratory animal center of Peking Union Medical College (PUMC). The conventional diet contains $41.47 \%$ carbohydrate, $14.42 \%$ fat, and $21.06 \%$ protein. The HFD contains $10 \%$ lard, $20 \%$ sucrose, $1 \%$ Choline chloride, $2.5 \%$ cholesterol, and $66.5 \%$ conventional diet.

\subsection{Model Establishment of T2DM, Grouping, and Sample} Collection. After adaptive feeding for one week, the rats were divided into five groups of ten rats in each group as follows: group 1, normal control rats; group 2, T2DM control rats; group 3, T2DM rats supplemented with $25 \mu \mathrm{g} / \mathrm{kg}$ CrPic; group 4, T2DM rats supplemented with $50 \mu \mathrm{g} / \mathrm{kg}$ CrPic; and group 5, T2DM rats supplemented with $100 \mu \mathrm{g} / \mathrm{kg}$ CrPic. Ten rats of normal control group were fed with conventional diet. Forty rats of the other four groups were fed with HFD. After four weeks, the forty rats of the other four groups were injected intraperitoneally with low dose $(30 \mathrm{mg} / \mathrm{kg})$ of STZ (Sigma) which was prepared in sterile citrate buffer (w/v: $2 \%)$. Ten rats of the normal control group were injected intraperitoneally with the same volume of sterile citrate buffer. After $72 \mathrm{~h}$, the peripheral blood glucose level in tails of rats was detected by the Precision Xtra blood glucose monitoring system (Alameda, CA, USA). The model was successfully established if the blood glucose of the rats was over $16.7 \mathrm{mmol} / \mathrm{L}$ and polyuria and polydipsia appeared in these rats. After the levels of blood glucose stably were maintained for one week, the blood glucose and serum insulin concentrations of the rats in each group were determined before supplementing CrPic (Spectrum chemical \& laboratory products, China). The rats of groups 3, 4, and 5 were supplemented with $\mathrm{CrPic}$ by gavage with equal volume of normal saline (NS; $0.9 \% \mathrm{NaCl}$ ) that contained different concentration of $\mathrm{CrPic}$ at every morning for 15 weeks. The rats of groups 1 and 2 were only supplemented with equal volume of NC that did not contain CrPic. After last time of CrPic administration, the rats were fasted but were not prohibited from drinking water for $12 \mathrm{~h}$. The weight change and food intake of the rats in each group were monitored once a week and the behaviors and mental state of all the rats were observed during the whole experiment. Finally, all the rats were decapitated and the blood and the fat from the abdomen were collected for biochemical analyses. The pancreas was collected for hematoxylin-eosin (HE) staining.

2.3. Histopathological Analysis. The histopathological examinations of the pancreatic sections of the rats in each group were performed by standard histological techniques with HE staining. The collected pancreas tissue was fixed in $10 \%$ buffered formalin and embedded in paraffin and sections of pancreatic tissue were deparaffinized and stained with $\mathrm{HE}$. The pathological changes of the lesion and its vicinity were observed by the light microscopy.

2.4. Determination of Serum Markers of T2DM. The detection of serum nitric oxide (NO) level was detected by using the Nitric Oxide Colorimetric Assay Kit (Nanjing Jiancheng Bioengineering Institute, China) following manufacturer's instruction. The serum insulin level was determined by using the radioimmunoassay kit (China Institute of Atomic Energy) according to the procedure described by the manufacturer. The serum levels of glycosylated hemoglobin (HbAlC), advanced glycation end products (AGES), adiponectin (APN), and apelin were assayed with an ELISA kit (R\&D System, Minneapolis, MN).

2.5. Determination of mRNA Expression of APN and Apelin. The fat tissues obtained from abdomen were processed for RNA extraction. The TRIzol reagent (Invitrogen, Carlsbad, CA) was used to extract total RNAs for analyzing the mRNA expression of APN and apelin mRNA. The cDNA was synthesized out of the total RNA with a cDNA synthesis kit (Promega, Southampton, UK). The $\beta$-actin was regarded as control. The primer sequences of $A P N$, apelin, and $\beta$ actin were shown in Table 1. Real-time reverse transcription polymerase chain reaction (RT-PCR) was performed using a reverse transcription Kit (Promega, Southampton, UK) following the manufacturer's protocol. The reaction condition of the real-time RT-PCR was 40 cycles of $95^{\circ} \mathrm{C}$ for $15 \mathrm{~s}, 55^{\circ} \mathrm{C}$ for $15 \mathrm{~s}$, and $72^{\circ} \mathrm{C}$ for $15 \mathrm{~s}$. Relative quantification of gene expression was done using the comparative CT $\left(2^{-\Delta \Delta \mathrm{CT}}\right)$ method.

2.6. Statistical Analyses. The data was analyzed by using the SPSS 13.0 software. Comparisons among four experimental groups were analyzed by one-way analysis of variance (ANOVA) followed by Bonferroni's test to evaluate statistical difference between two groups. $P$ values less than 0.05 were defined as statistically significant. All data are presented as mean \pm standard deviation.

\section{Results}

3.1. Establishment of T2DM Rat Model. After injecting STZ for $72 \mathrm{~h}$, the average concentrations of blood glucose of the rats in groups 2 to 5 all reached $16.7 \mathrm{mmol} / \mathrm{L}$. Then, the levels 
TABLE 1: The primer sequences of the APN, apelin, and $\beta$-actin.

\begin{tabular}{lcc}
\hline Gene name & Forward primer & Reverse primer \\
\hline APN & CTG GAG AGA AGG GAG AGA AG & GCT GAA TGG TGA GTG ATA CA \\
Apelin & CTG CTC TGG CTC TCC TTG AC & ATG GGT CCC TTA TGG GAG AG \\
$\beta$-Actin & TCT TCC AGC CTT CCT TCC TG & TAG AGC CAC CAA TCC ACA CA \\
\hline
\end{tabular}

APN: adiponectin.

TABLE 2: The blood glucose and serum insulin concentrations of the rats after the blood glucose levels were stably maintained for one week.

\begin{tabular}{|c|c|c|c|c|c|}
\hline \multirow{2}{*}{ Group } & \multirow{2}{*}{$\mathrm{CN}$} & \multirow{2}{*}{$\mathrm{T} 2 \mathrm{DM}$} & \multicolumn{3}{|c|}{ CrPic groups } \\
\hline & & & $(25 \mu \mathrm{g} / \mathrm{kg})$ & $(50 \mu \mathrm{g} / \mathrm{kg})$ & $(100 \mu \mathrm{g} / \mathrm{kg})$ \\
\hline Blood glucose $(\mathrm{mmol} / \mathrm{L})$ & $4.63 \pm 1.02$ & $16.81 \pm 3.65^{*}$ & $17.52 \pm 2.76^{*}$ & $17.91 \pm 2.89^{*}$ & $18.03 \pm 1.15^{*}$ \\
\hline Serum insulin (mIU/L) & $16.88 \pm 0.83$ & $11.51 \pm 1.07^{*}$ & $10.75 \pm 1.23^{*}$ & $9.98 \pm 1.03^{*}$ & $11.96 \pm 0.74^{*}$ \\
\hline
\end{tabular}

CN: the normal control group, group 1; T2DM: T2DM (type II diabetes mellitus) control group, group 2; CrPic groups: group 3 ( $25 \mu \mathrm{g} / \mathrm{kg})$, group 4 (50 $\mu \mathrm{g} / \mathrm{kg}$ ), and group $5(100 \mu \mathrm{g} / \mathrm{kg})$; and ${ }^{*}$ compared with group $1, P<0.01$.

of blood glucose were stably maintained for one week. The blood glucose and serum insulin concentrations of the rats are shown in Table 2. Compared with group 1, the blood glucose and serum insulin concentrations of the rats in the other four groups were significantly higher, which indicated that the rats suffered T2DM induced by STZ. In addition, the apathetic state and behaviors of polyuria and polydipsia appeared in the rats of groups 2 to 5. Therefore, the T2DM rat model was successfully established.

3.2. Effects of Chromium Picolinate on Histopathology of Pancreas. The pancreatic sections stained with $\mathrm{HE}$ are shown in Figure 1. It showed that the rats in group 1 had normal pancreatic endocrine and exocrine architecture, acinar cells, and pancreatic islets. Moreover, there was no edema or inflammatory cells infiltration (Figure 1(a)). However, administration of STZ caused necrotic changes (karyolysis and disappearance of nucleus) of pancreatic islets, degeneration of acinar cells, and inflammatory cells infiltration in STZ-induced T2DM rats (Figure 1(b): group 2). After CrPic treatment, some improvements on the morphology of pancreas were observed. In the rats supplemented with $50 \mu \mathrm{g} / \mathrm{kg}$ (Figure 1(d): group 4) and $100 \mu \mathrm{g} / \mathrm{kg} \mathrm{CrPic}$ (Figure 1(e): group 5), there was completed pancreatic cells structure and no inflammatory cell infiltration was observed. In the rats supplemented with $25 \mu \mathrm{g} / \mathrm{kg}$ CrPic (Figure 1(c): group 3), the improvement on the morphology of pancreas was not significant compared with the rats supplemented with $50 \mu \mathrm{g} / \mathrm{kg}$ and $100 \mu \mathrm{g} / \mathrm{kg}$ CrPic.

3.3. Effects of Chromium Picolinate on Serum Markers. Compared with group 1, NO and insulin level of the rats in group 2 were significantly decreased $(P<0.01)$. After $\mathrm{CrPic}$ treatment, the NO and insulin levels of the rats in groups $4(P<$ $0.05)$ and $5(P<0.01)$ were significantly increased compared with group 2 . The $\mathrm{NO}$ and insulin level of the rats in group 3 were also higher than those in group 2, but the statistical difference was not significant. The dose dependent effect was observed in the effect of CrPic on $\mathrm{NO}$ and insulin level of STZ-induced T2DM rats which were raised with the increase of the dose of CrPic. The NO and insulin level of the rats in group 5 were very close to group 1 (Figures 2(a) and 2(b)).

According to Figures 2(c), 2(d), 2(e), and 2(f), the levels of $\mathrm{HbA1C}$, AGES, APN, and apelin of rats in group 2 were significantly higher than those in group $1(P<0.01)$. After CrPic treatment, the levels of HbA1C, AGES, APN, and apelin of the rats in groups $4(P<0.05)$ and $5(P<0.01)$ were significantly reduced compared with group 2 . The dose dependent effect was also observed in the effect of CrPic on levels of HbA1C, AGES, APN, and apelin of STZ-induced T2DM rats which were reduced with the decrease of the dose of CrPic. The decrease of the levels of HbA1C, AGES, APN, and apelin of the rats in group 3 was not significant compared with group 2. The levels of HbA1C, AGES, APN, and apelin level of the rats in group 5 were almost close to group 1.

3.4. Effects of Chromium Picolinate on the mRNA Expression of APN and Apelin. The mRNA expression levels of APN and apelin were significantly higher in group 2 than those in group $1(P<0.05)$. After CrPic treatment, the mRNA expression levels of $A P N$ and apelin of the rats in groups 3 $(P<0.05), 4(P<0.01)$, and $5(P<0.01)$ were significantly decreased compared with group 2 (Figure 3 ).

3.5. The Weight Change and Food Intake of the Rats. The weight change and food intake of the rats in each group during the whole experiment are shown in Table 3. Compared with group 1, the weight changes of the rats in the other four groups were significantly less while the food intake was significantly more. After the administration of $\mathrm{CrPic}$, the weight changes and food intake of the STZ-induced T2DM rats in groups 3 to 5 were gradually close to group 1 with the increase of the dose of CrPic.

\section{Discussion}

T2DM is a metabolism syndrome with multifactors and involved multiorgans, which is characterized by chronic high blood glucose. Macroangiopathy was usually seen as the complication in the patients with diabetes. The macroangiopathy 


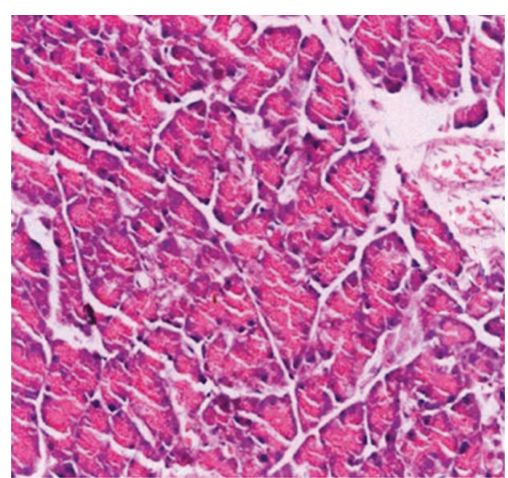

(a)

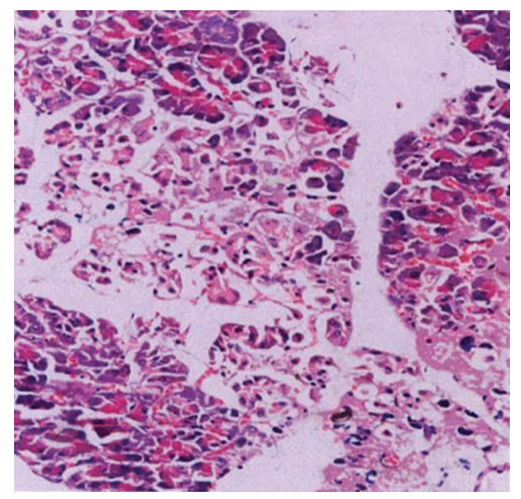

(c)

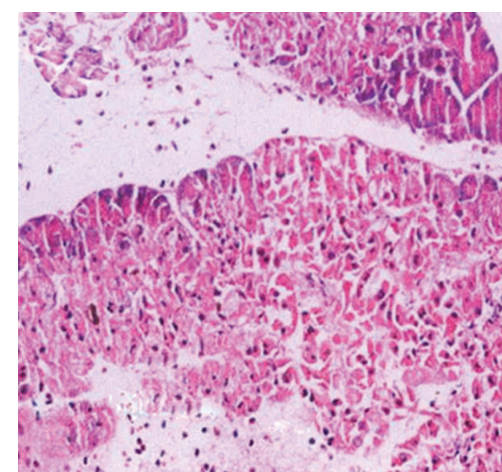

(b)

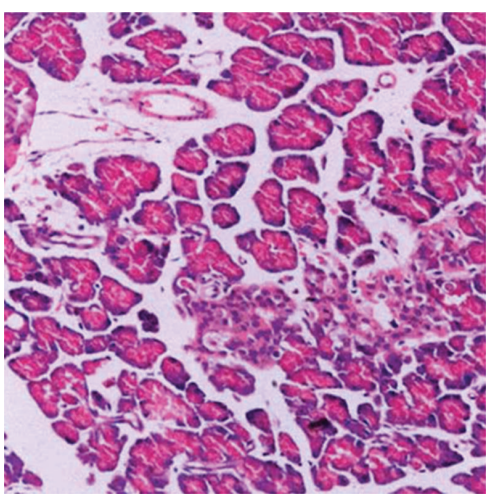

(d)

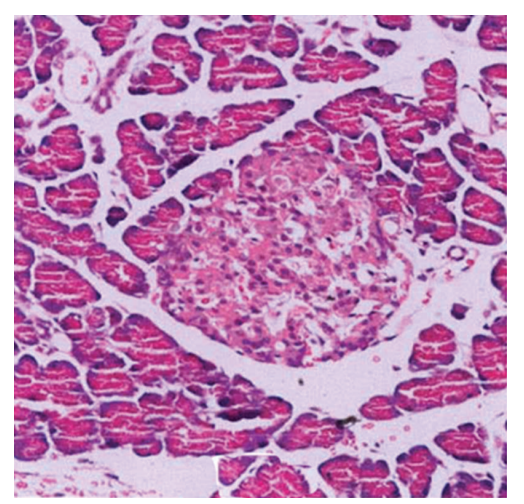

(e)

FIGURE 1: The HE staining of pancreas (HE 400x) in five groups. (a) Group 1, normal control group; (b) group 2, T2DM (type II diabetes mellitus) control group; (c) group 3, $25 \mu \mathrm{g} / \mathrm{kg} \mathrm{CrPic}$ group; (d) group 4, $50 \mu \mathrm{g} / \mathrm{kg} \mathrm{CrPic}$ group; and (e) group 5, $100 \mu \mathrm{g} / \mathrm{kg} \mathrm{CrPic}$ group.

is one of the main reasons of death and disability [23, 24]. In addition, the pathogenesis of T2DM was very complicated and associated with insulin resistance and secretion deficiency [25, 26], inflammatory reaction [27], and oxidative stress [28]. Therefore, it is critical to find the suitable therapy of treating T2DM and macroangiopathy based on the pathogenesis of T2DM.

CrPic is organic chromium compound with small molecular weight and low toxicity. It is promising in therapy application because it can increase the activity of insulin and slow down the development of diabetes mellitus [29]. In the present study, the T2DM model was induced by STZ; STZ liberates toxic amounts of nitric oxide that inhibits aconitase activity and participates in DNA damage [30]. As a result of the STZ action, $\beta$-cells undergo destruction by necrosis. After CrPic supplement, the inflammatory cells infiltration was reduced or appeared and the function of $\beta$-cells was recovered.

For the levels of serum markers of T2DM, the results provided evidence for the ability of $\mathrm{CrPic}$ in regulating the levels of NO, insulin, HbA1C, AGES, APN, and apelin. T2DM frequently results from progressive failure of pancreatic $\beta$ cell function in the presence of chronic insulin resistance [31]. Some previous studies have reported that CrPic supplementation could increase insulin sensitivity in subjects with type 2 diabetes $[22,32,33]$. The secretion of insulin comes 
TABLE 3: The weight change and food intake of the rats in each group during the whole experiment.

\begin{tabular}{|c|c|c|c|c|c|}
\hline \multirow{2}{*}{ Group } & \multirow{2}{*}{$\mathrm{CN}$} & \multirow{2}{*}{$\mathrm{T} 2 \mathrm{DM}$} & \multicolumn{3}{|c|}{ CrPic groups } \\
\hline & & & $(25 \mu \mathrm{g} / \mathrm{kg})$ & $(50 \mu \mathrm{g} / \mathrm{kg})$ & $(100 \mu \mathrm{g} / \mathrm{kg})$ \\
\hline Weight change (g/week) & $397.80 \pm 21.74$ & $349.551 \pm 13.25^{*}$ & $357.32 \pm 12.93^{*}$ & $357.11 \pm 14.50^{*}$ & $360.03 \pm 21.01^{*}$ \\
\hline Food intake (g/day) & $16 \pm 3$ & $42 \pm 7^{*}$ & $37 \pm 5^{*}$ & $29 \pm 4^{*}$ & $27 \pm 7^{*}$ \\
\hline
\end{tabular}

CN: the normal control group, group 1; T2DM: T2DM (type II diabetes mellitus) control group, group 2; CrPic groups: group 3 ( $25 \mu \mathrm{g} / \mathrm{kg})$, group 4 (50 $\mu \mathrm{g} / \mathrm{kg})$, and group $5(100 \mu \mathrm{g} / \mathrm{kg})$; and ${ }^{*}$ compared with group $1, P<0.01$.

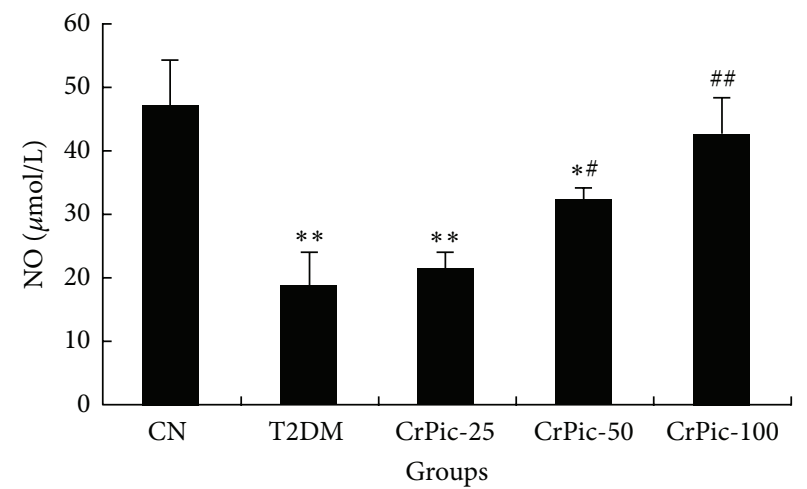

(a)

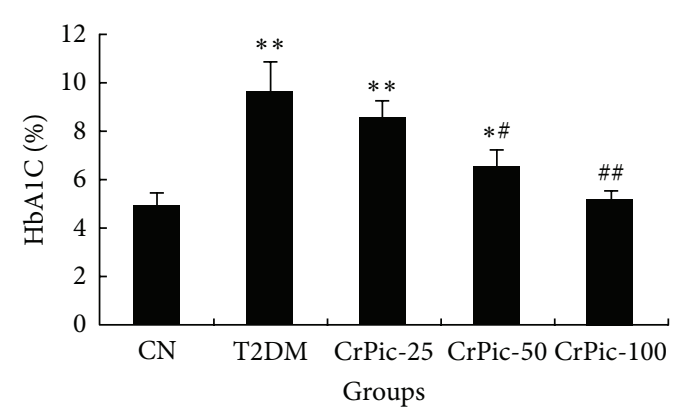

(c)

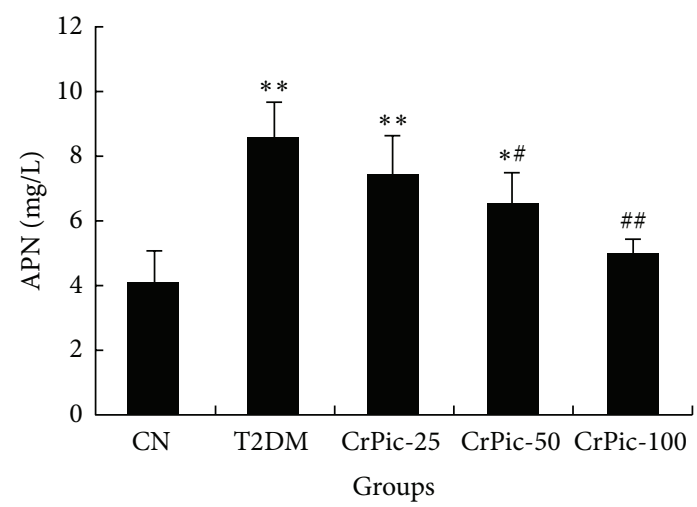

(e)

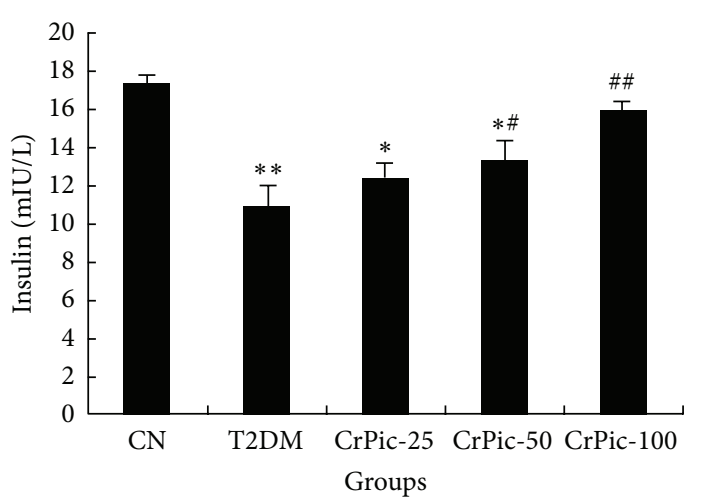

(b)

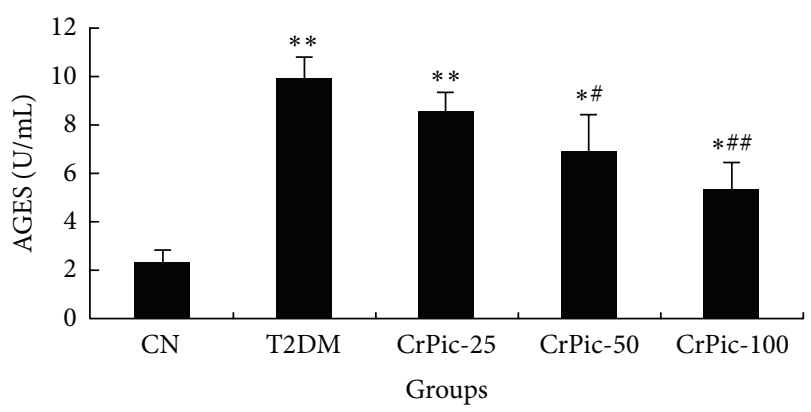

(d)

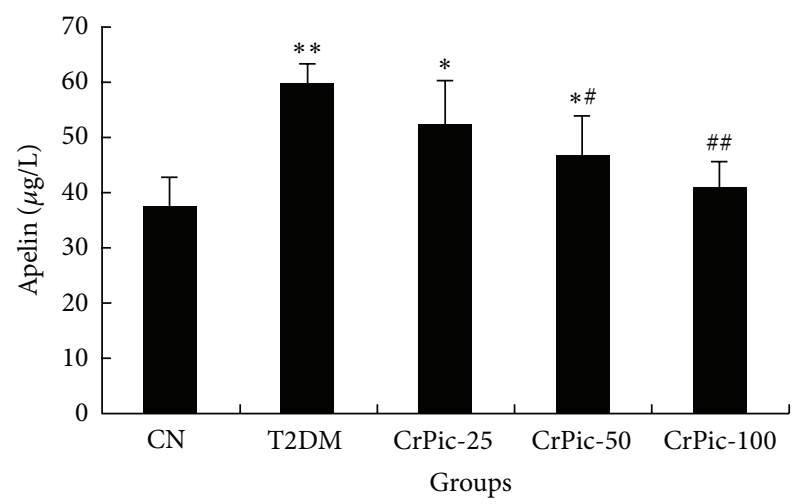

(f)

FIGURE 2: The effect of CrPic on the levels of serum NO (a), insulin (b), HbAlC (c), AGES (d), APN (e), and apelin (f). CN: the normal control group, group 1; T2DM: T2DM (type II diabetes mellitus) control group, group 2; CrPic-25: group 3 (25 $\mu \mathrm{g} / \mathrm{kg}$ ); CrPic-50: group 4 (50 $\mu \mathrm{g} / \mathrm{kg}$ ); and CrPic-100: group $5(100 \mu \mathrm{g} / \mathrm{kg}) .{ }^{* *} P<0.01$ compared with group $1 ;{ }^{*} P<0.05$ compared with group $1 ;{ }^{\# \#} P<0.01$ compared with group 2 ; and ${ }^{\#} P<0.05$ compared with group 2 . 


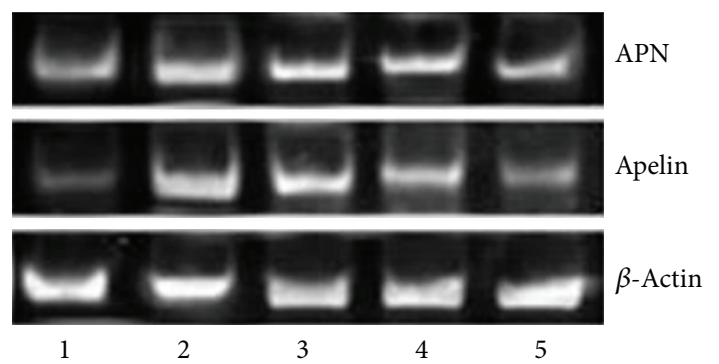

(a)

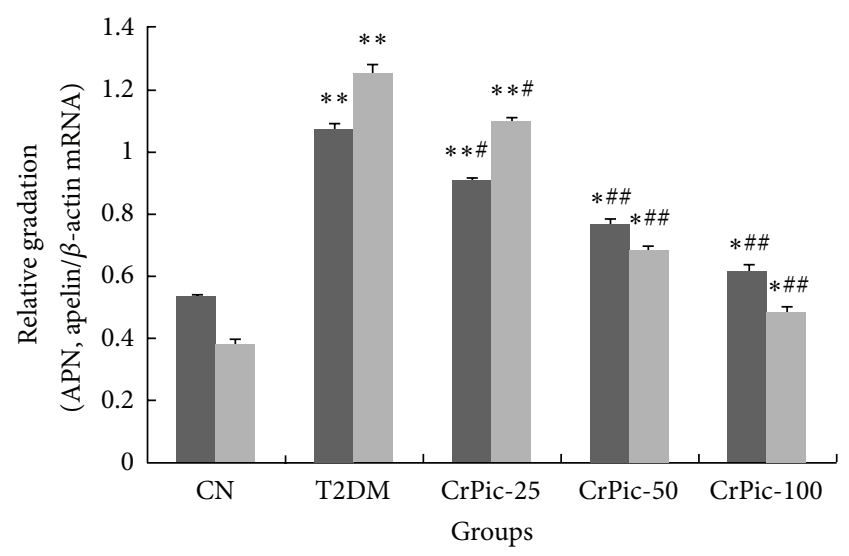

APN

Apelin

FIGURE 3: The mRNA expression levels of APN and apelin in each group. (a) Agarose gel electrophoresis of RT-PCR products. (b) The histogram of relative mRNA expression levels of APN and apelin compared with $\beta$-actin. CN: the normal control group, group 1; T2DM: T2DM (type II diabetes mellitus) control group, group 2; CrPic-25: group 3 (25 $\mu \mathrm{g} / \mathrm{kg}$ ); CrPic-50: group 4 (50 $\mu \mathrm{g} / \mathrm{kg}$ ); and CrPic-100: group $5(100 \mu \mathrm{g} / \mathrm{kg}) .{ }^{* *} P<0.01$ compared with group $1 ;{ }^{*} P<0.05$ compared with group $1 ;{ }^{\# \#} P<0.01$ compared with group 2 ; and ${ }^{\#} P<0.05$ compared with group 2.

from $\beta$-cell. Therefore, we speculated that the level of insulin was raised after $\mathrm{CrPic}$ treatment due to the increase of insulin sensitivity in T2DM rats and the improvement of $\beta$-cell function. In addition, $\mathrm{HbAlC}$, as another indicator of diabetes, is the product of hemoglobin and glucose in the red blood cells and it can be used as the reference standard of average blood glucose level. Previous study showed that $1 \%$ decrease of the $\mathrm{HbA1C}$ resulted in $21 \%$ decrease in diabetes related end-point event, and the risk of the diabetes related death and myocardial infarct will decrease $21 \%$ and $14 \%$, respectively [34]. After reconfiguration, the $\mathrm{HbA1C}$ can transform into AGES, so it is positively related to AGES. Then AGES can interact with its receptor and multiple cytokines and growth factors will be produced. Some articles reported that AGES could lead to vascular hyperplasia and accelerate the atherosclerosis $[35,36]$. Furthermore, the increase of AGES level can result in decreasing level of NO. Serum NO is one of the inflammatory cytokines. Some clinical and epidemiological studies suggest that some processes related to lowgrade inflammation may be relevant to diabetic micro- and macroangiopathy $[37,38]$. Thus, the macroangiopathy was associated with the levels of serum NO. In this study, the decrease of NO level in STZ-induced T2DM rats might cause the change of angiotasis and then the macroangiopathy happened. The efficacy of CrPic on treating macroangiopathy was proved by the increase of serum NO level after CrPic treatment. Moreover, the changes of these markers were all dose dependent. In summary, CrPic may affect macroangiopathy in T2DM by increasing the NO level and decreasing the HbA1C and AGES level.

APN and apelin are the cytokines secreted by adipocytes. It was reported that the increase of the APN was observed in the patients with coronary artery disease and early atherosclerosis $[39,40]$. In this study, the STZ injection with HFD will induce the mRNA expression of APN and apelin; it indicated that the macroangiopathy might occur with the T2DM. With the treatment of CrPic, the expression of the two genes was recovered back to normal level. These results indicated that the effect of CrPic on macroangiopathy in T2DM may be associated with the regulation of the lipid metabolism.

Furthermore, the weight change and food intake were also improved after CrPic treatment. The weight gain of rats was attenuated by STZ administration. It may be caused by the decrease of insulin. The dysregulation of insulin on blood glucose may affect the conversion of glucose and lipid metabolism [41]. Thus, the weight gain was decreased after STZ administration. Then, the weight gain was recovered to normal with the CrPic treatment. Based on the results of this study, the food intake was decreased after CrPic supplement. Thus, we inferred that the mechanism of the changes of weight gain in T2DM rats might be related to the effect of CrPic on the serum and fat markers such as NO, insulin, HbA1C, AGES, APN, and apelin.

\section{Conclusion}

In conclusion, $\mathrm{CrPic}$ can recover the function of $\beta$-cells and alleviate macroangiopathy in STZ-induced T2DM rats. Further studies were required to verify the results of this study.

\section{Conflict of Interests}

The authors declare that there is no conflict of interests regarding the publication of this paper. 


\section{Acknowledgments}

This paper was supported by Changning District Diabetes Special Disease-Funded Project (2010301001) and Shanghai Municipal Health Bureau Project (20124248).

\section{References}

[1] J. Hjelmesaeth, A. Hartmann, T. Leivestad et al., "The impact of early-diagnosed new-onset post-transplantation diabetes mellitus on survival and major cardiac events," Kidney International, vol. 69, no. 3, pp. 588-595, 2006.

[2] Y. M. Choi, P. Ilankumaran, H. W. Kim et al., "Chroman carboxylic acid derivatives for the treatment of diabetes and lipid disorders," Google Patents, 2008.

[3] U. Nöthlings, H. Boeing, G. Maskarinec et al., "Food intake of individuals with and without diabetes across different countries and ethnic groups," European Journal of Clinical Nutrition, vol. 65, no. 5, pp. 635-641, 2011.

[4] E. Ritz and S. R. Orth, "Nephropathy in patients with type 2 diabetes mellitus," The New England Journal of Medicine, vol. 341, no. 15, pp. 1127-1133, 1999.

[5] L. Krugner-Higby, M. Shadoan, C. Carlson et al., “Type 2 diabetes mellitus, hyperlipidemia, and extremity lesions in California mice (Peromyscus californicus) fed commercial mouse diets," Comparative Medicine, vol. 50, no. 4, pp. 412-418, 2000.

[6] M. Yamamoto, G. Egusa, M. Okubo, and M. Yamakido, "Dissociation of microangiopathy and macroangiopathy in patients with type 2 diabetes," Diabetes Care, vol. 21, no. 9, pp. 1451-1454, 1998.

[7] K. Tsukada, E. Sekizuka, C. Oshio, and H. Minamitani, "Direct measurement of erythrocyte deformability in diabetes mellitus with a transparent microchannel capillary model and highspeed video camera system," Microvascular Research, vol. 61, no. 3, pp. 231-239, 2001.

[8] S. B. Harris, J. Ekoé, Y. Zdanowicz, and S. Webster-Bogaert, "Glycemic control and morbidity in the Canadian primary care setting (results of the diabetes in Canada evaluation study)," Diabetes Research and Clinical Practice, vol. 70, no. 1, pp. 90-97, 2005.

[9] K. Strojek, "Features of macrovascular complications in type 2 diabetic patients," Acta Diabetologica, vol. 40, no. 2, pp. S334S337, 2003.

[10] R. A. Anderson, N. Cheng, N. A. Bryden et al., "Elevated intakes of supplemental chromium improve glucose and insulin variables in individuals with type 2 diabetes," Diabetes, vol. 46, no. 11, pp. 1786-1791, 1997.

[11] Q. Zhao, X. Hu, Q. Guo et al., "Physicochemical properties and regulatory effects on $\mathrm{db} / \mathrm{db}$ diabetic mice of $\beta$-glucans extracted from oat, wheat and barley," Food Hydrocoll, vol. 37, pp. 60-68, 2014.

[12] K. H. Sumrall and J. B. Vincent, "Is glucose tolerance factor an artifact produced by acid hydrolysis of low-molecular-weight chromium-binding substance?" Polyhedron, vol. 16, no. 23, pp. 4171-4177, 1997.

[13] N. Sreejayan, F. Dong, M. R. Kandadi, X. Yang, and J. Ren, "Chromium alleviates glucose intolerance, insulin resistance, and hepatic ER stress in obese mice," Obesity, vol. 16, no. 6, pp. 1331-1337, 2008.

[14] M. Toghyani, M. Toghyani, M. Shivazad, A. Gheisari, and R. Bahadoran, "Chromium supplementation can alleviate the negative effects of heat stress on growth performance, carcass traits, and meat lipid oxidation of broiler chicks without any adverse impacts on blood constituents," Biological Trace Element Research, vol. 146, no. 2, pp. 171-180, 2012.

[15] C. M. Davis and J. B. Vincent, "Chromium in carbohydrate and lipid metabolism," Journal of Biological Inorganic Chemistry, vol. 2, no. 6, pp. 675-679, 1997.

[16] M. J. Tsapakos and K. E. Wetterhahn, "The interaction of chromium with nucleic acids," Chemico-Biological Interactions, vol. 46, no. 2, pp. 265-277, 1983.

[17] A. Mikalsen, J. Alexander, H. Wallin, M. Ingelman-Sundberg, and R. A. Andersen, "Reductive metabolism and protein binding of chromium(VI) by $\mathrm{P} 450$ protein enzymes," Carcinogenesis, vol. 12, no. 5, pp. 825-831, 1991.

[18] H. C. Lukaski, “Chromium as a supplement," Annual Review of Nutrition, vol. 19, pp. 279-302, 1999.

[19] G. J. Ryan, N. S. Wanko, A. R. Redman, and C. B. Cook, "Chromium as adjunctive treatment for type 2 diabetes," Annals of Pharmacotherapy, vol. 37, no. 6, pp. 876-885, 2003.

[20] M.-Q. Wang, H. Li, Y.-D. He, C. Wang, W. Tao, and Y. Du, "Efficacy of dietary chromium (III) supplementation on tissue chromium deposition in finishing pigs," Biological Trace Element Research, vol. 148, no. 3, pp. 316-321, 2012.

[21] W. T. Cefalu, J. Rood, P. Pinsonat et al., "Characterization of the metabolic and physiologic response to chromium supplementation in subjects with type 2 diabetes mellitus," Metabolism: Clinical and Experimental, vol. 59, no. 5, pp. 755-762, 2010.

[22] J. Martin, Z. Q. Wang, X. H. Zhang et al., "Chromium picolinate supplementation attenuates body weight gain and increases insulin sensitivity in subjects with type 2 diabetes," Diabetes Care, vol. 29, no. 8, pp. 1826-1832, 2006.

[23] S. Dronavalli, I. Duka, and G. L. Bakris, "The pathogenesis of diabetic nephropathy," Nature Clinical Practice Endocrinology \& Metabolism, vol. 4, no. 8, pp. 444-452, 2008.

[24] M. P. Hermans, S. A. Ahn, and M. F. Rousseau, "The multifaceted outcomes of conjunct diabetes and cardiovascular familial history in type 2 diabetes," Journal of Diabetes and its Complications, vol. 26, no. 3, pp. 187-194, 2012.

[25] E. Nylander and S. Svartholm, "Self care activities of patients with Diabetes Mellitus Type 2 in Ho Chi Minh City," 2010.

[26] S. Wild, G. Roglic, A. Green, R. Sicree, and H. King, "Global prevalence of diabetes: estimates for the year 2000 and projections for 2030," Diabetes Care, vol. 27, no. 5, pp. 1047-1053, 2004.

[27] T. Fujita, S. Hemmi, M. Kajiwara et al., "Complement-mediated chronic inflammation is associated with diabetic microvascular complication," Diabetes/Metabolism Research and Reviews, vol. 29, no. 3, pp. 220-226, 2013.

[28] P. Roesen, P. Ferber, and D. Tschoepe, "Macrovascular disease in diabetes: current status," Experimental and Clinical Endocrinology and Diabetes, vol. 109, supplement 2, pp. S474-S486, 2001.

[29] K. Sahin, M. Tuzcu, C. Orhan et al., "Anti-diabetic activity of chromium picolinate and biotin in rats with type 2 diabetes induced by high-fat diet and streptozotocin," British Journal of Nutrition, vol. 110, no. 2, pp. 197-205, 2013.

[30] T. Szkudelski, "The mechanism of alloxan and streptozotocin action in B cells of the rat pancreas," Physiological Research, vol. 50, no. 6, pp. 537-546, 2001.

[31] T. A. Buchanan, A. H. Xiang, R. K. Peters et al., "Preservation of pancreatic $\beta$-cell function and prevention of type 2 diabetes by pharmacological treatment of insulin resistance in high-risk Hispanic women," Diabetes, vol. 51, no. 9, pp. 2796-2803, 2002. 
[32] D.-S. Kim, T.-W. Kim, and J.-S. Kang, "Chromium picolinate supplementation improves insulin sensitivity in Goto-Kakizaki diabetic rats," Journal of Trace Elements in Medicine and Biology, vol. 17, no. 4, pp. 243-247, 2004.

[33] D.-S. Kim, T.-W. Kim, I.-K. Park, J.-S. Kang, and A.-S. Om, "Effects of chromium picolinate supplementation on insulin sensitivity, serum lipids, and body weight in dexamethasonetreated rats," Metabolism: Clinical and Experimental, vol. 51, no. 5, pp. 589-594, 2002.

[34] I. M. Stratton, A. I. Adler, H. A. W. Neil et al., "Association of glycaemia with macrovascular and microvascular complications of type 2 diabetes (UKPDS 35): prospective observational study," British Medical Journal, vol. 321, no. 7258, pp. 405-412, 2000.

[35] S. Del Turco and G. Basta, "An update on advanced glycation endproducts and atherosclerosis," BioFactors, vol. 38, no. 4, pp. 266-274, 2012.

[36] J. Nam and R. Fettiplace, "Optimal electrical properties of outer hair cells ensure cochlear amplification," PLoS ONE, vol. 7, no. 11, Article ID e50572, 2012.

[37] V. Jakuš, E. Sándorová, J. Kalninová, and B. Krahulec, “Monitoring of glycation, oxidative stress and inflammation in relation to the occurrence of vascular complications in patients with type 2 diabetes mellitus," Physiological Research, 2014.

[38] D. Zozuliñska and B. Wierusz-Wysocka, "Hyperglycaemia and inflammation are culpritis of late diabetic complications," Archives of Medical Science, vol. 1, pp. 115-118, 2005.

[39] A. Hirata, K. Kishida, H. Nakatsuji, H. Kobayashi, T. Funahashi, and I. Shimomura, "High serum Clq-adiponectin/total adiponectin ratio correlates with coronary artery disease in Japanese type 2 diabetics," Metabolism: Clinical and Experimental, vol. 62, no. 4, pp. 578-585, 2013.

[40] Y. Xiao, L. Yao, X. Li et al., "Relationship of adipocyte fatty acidbinding protein to adiponectin ratio with femoral intima-media thickness and endothelium-dependent vasodilation in patients with newly-diagnosed type 2 diabetes mellitus," Zhonghua Yi Xue Za Zhi, vol. 90, no. 4, pp. 231-235, 2010.

[41] A. R. Saltiel and C. R. Kahn, "Insulin signalling and the regulation of glucose and lipid metabolism," Nature, vol. 414, no. 6865, pp. 799-806, 2001. 


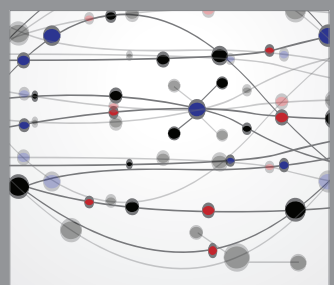

The Scientific World Journal
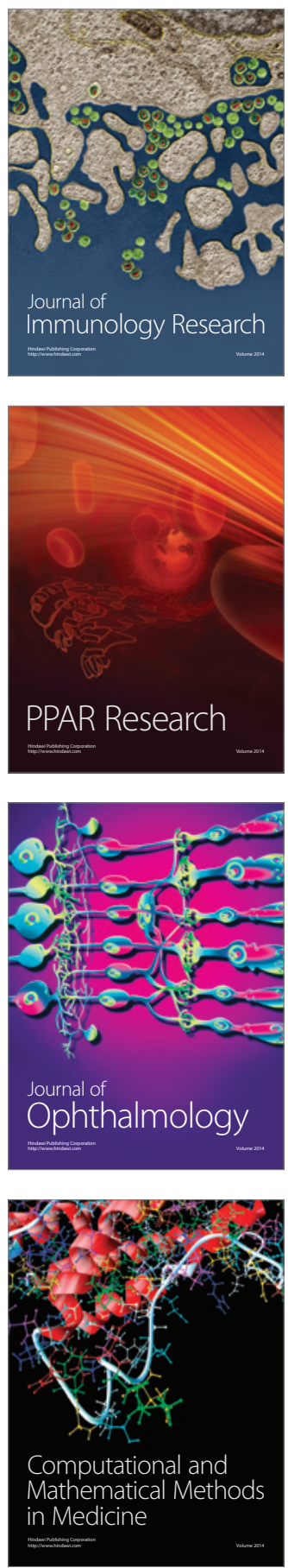

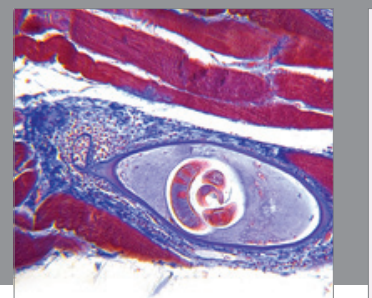

Gastroenterology

Research and Practice
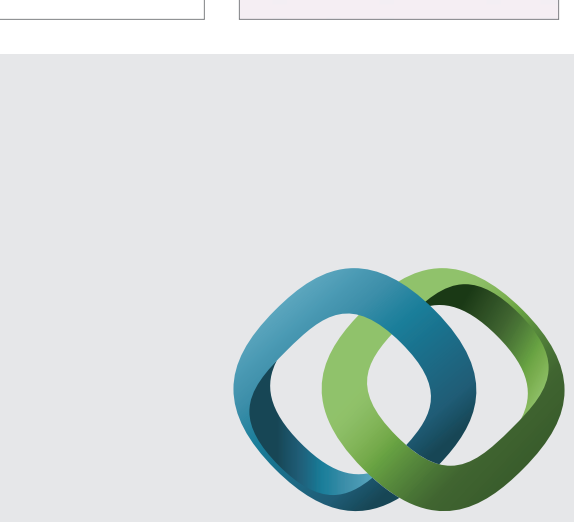

\section{Hindawi}

Submit your manuscripts at

http://www.hindawi.com
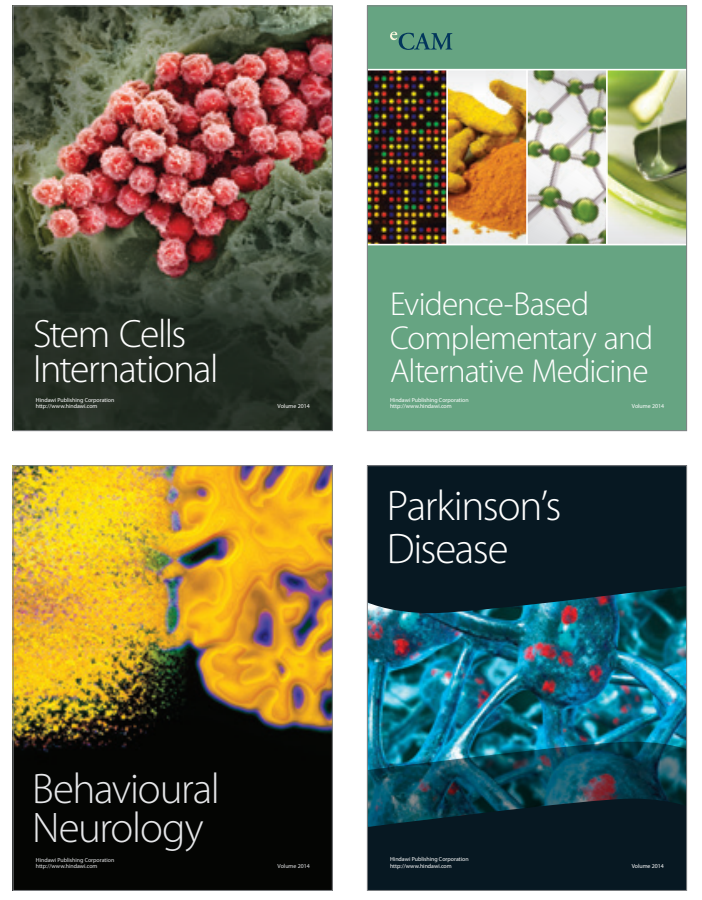
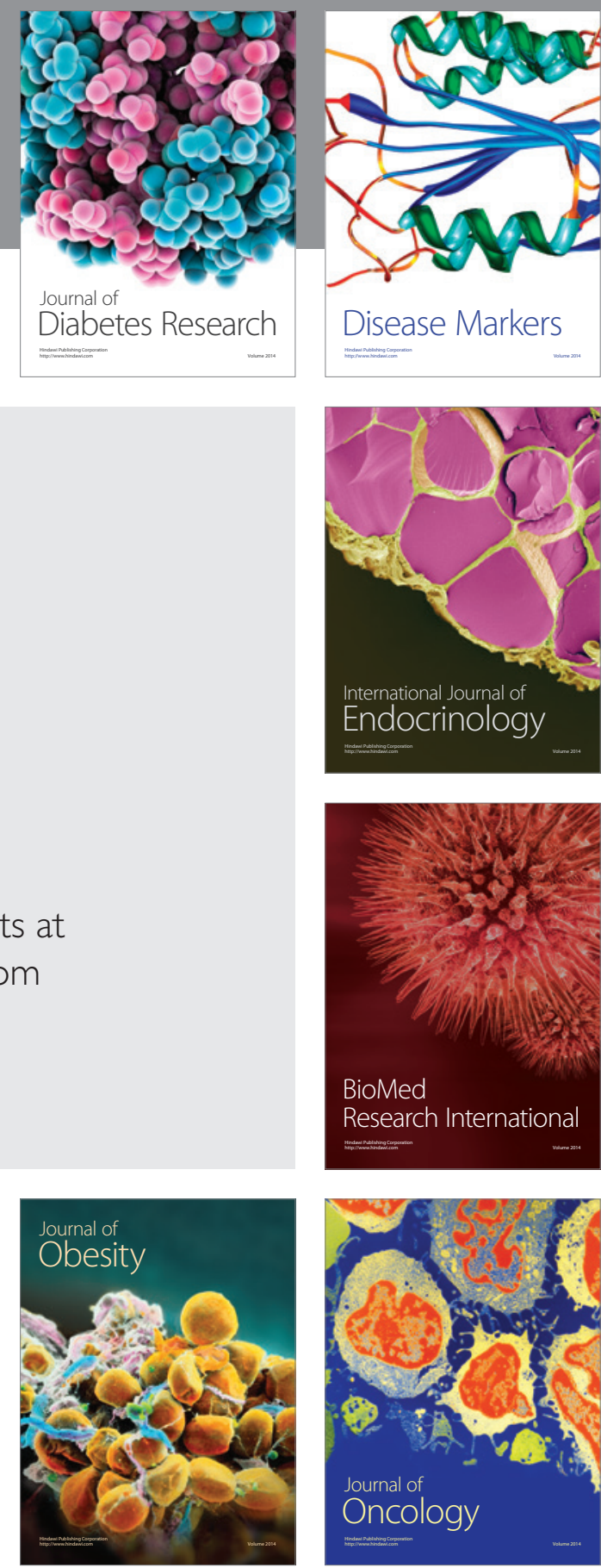

Disease Markers
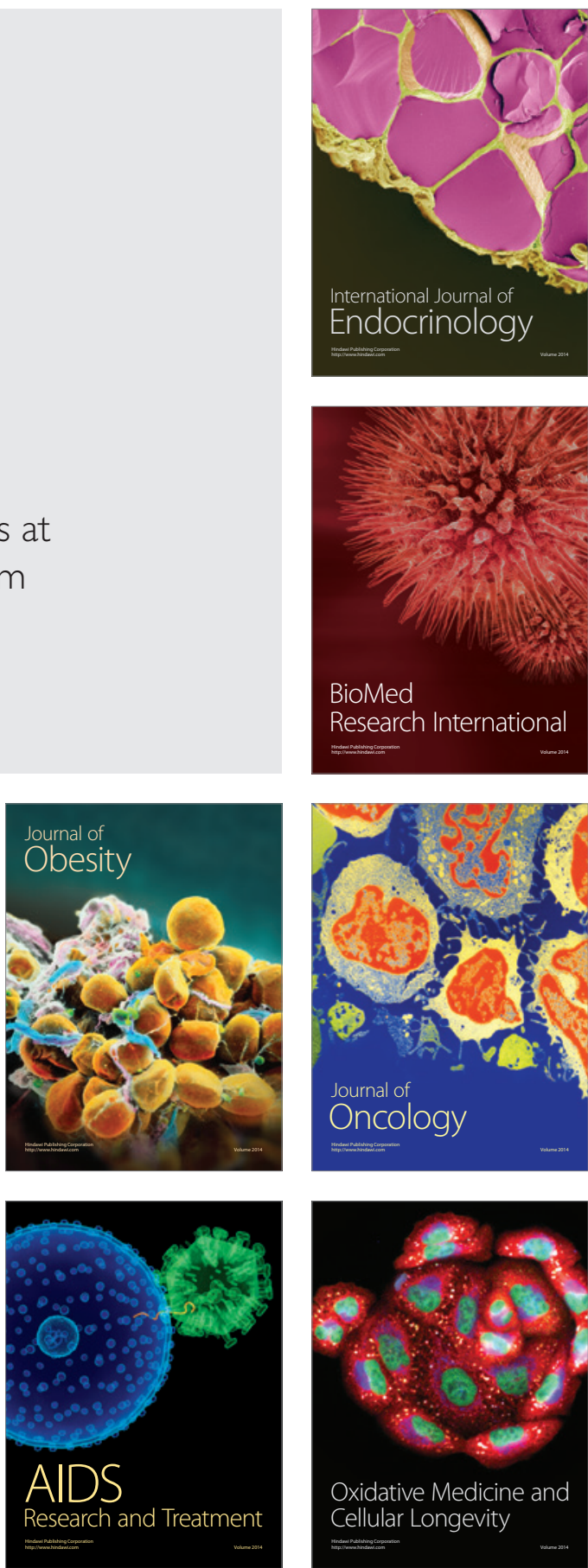